\title{
Improving adhesion of copper/epoxy joints by pulsed laser ablation
}

\author{
Edwin Hernandez ${ }^{\mathrm{a}}$, Marco Alfano ${ }^{\mathrm{b}, *}$, Gilles Lubineau ${ }^{\mathrm{a}}$, Ulrich Buttner ${ }^{\mathrm{c}}$ \\ ${ }^{a}$ King Abdullah University of Science and Technology, Physical Sciences and Engineering Division, \\ COHMAS Laboratory, Thuwal 23955-6900, Saudi Arabia \\ ${ }^{b}$ Department of Mechanical, Energy and Management Engineering, University of Calabria, Via P. \\ Bucci 44C, 87036 Rende (CS), Italy \\ ${ }^{c}$ King Abdullah University of Science and Technology, Computer, Electrical and Mathematical Sciences \\ and Engineering Division, Electrical Engineering Department, Thuwal 23955-6900, Saudi Arabia
}

\begin{abstract}
The purpose of the present work is to survey the effect of pulsed laser ablation on copper substrates (CuZn40) deployed for adhesive bonding. Surface pre-treatment was carried using an Yb-fiber laser beam. Treated surfaces were probed using Scanning Electron Microscopy (SEM) and X-Ray Photoelectron Spectroscopy (XPS). The mechanical performance of CuZn40/epoxy bonded joints was assessed using the T-peel test coupon. In order to resolve the mechanisms of failure and adhesive penetration within surface asperities induced by the laser treatment, fracture surfaces were surveyed using SEM. Finite element simulations, based on the use of the cohesive zone model of fracture, were carried out to evaluate the variation of bond toughness. Results indicated that the laser ablation process effectively modifies surface morphology and chemistry and enables enhanced mechanical interlocking and cohesive failure within the adhesive layer. Remarkable improvements of apparent peel energy and bond toughness were observed with respect to control samples with sanded substrates.
\end{abstract}

Keywords: laser ablation, copper alloy, epoxy, interlocking

\footnotetext{
${ }^{*}$ Corresponding author. Tel.: +39-0984-494156

Email address: marco.alfano@unical.it (Marco Alfano)
} 


\section{Introduction and motivation}

Adhesive bonding is a suitable joining technique for the fabrication of lightweight structures, especially those requiring different materials (e.g. metals and composites) to be bonded together $[1,2]$.

However, adhesive joints show a rich array of failure mechanisms which makes their optimization quite complex. For instance, interfacial fracture, which is usually the result of improper surface preparation, is a very widespread mechanism of failure in bonded structures. Indeed, fabrication of reliable adhesive bonds does not solely depend on joint design and adhesive type, but it strongly relies on the preparation of the mating surfaces $[1,2]$. As a matter of fact, yield and reliability of adhesive bonds are strongly affected by interfacial adhesion and appropriate surface preparation techniques are then needed.

Extensive studies have been performed in order to improve debonding resistance through the development of surface pre-treatments which enable the formation of appropriate surface morphology and chemistry $[1,2,3]$. In general terms, surface pretreatments aim to promote intermolecular interactions at the adhesive/substrate interface as well as mechanical interlocking of the adhesive within surface asperities.

Current surface preparation techniques include mechanical abrasion by means of sanding or grit blasting [4]. However, limited repeatability and potential surface contamination may reduce the overall bond performance. There is a wide range of alternatives that can be undertaken, among the most sophisticated it is worth mentioning acid anodizing, which certainly plays a major role in the fabrication of bonded connections for the aerospace industry [5]. Yet, chemical treatments create a large volume of hazardous wastes and pose a greater risk to the environment and human health, as a result they have been progressively subjected to a stricter control regime by governmental organizations [6].

It follows that new surface preparation methods, which hold the promise of improved 
repeatability, costs reduction and waste minimization are becoming increasingly important. Alternative cleaner procedures include the use of solid state or fiber pulsed laser ablation (PLA) [7]. PLA relies on the use of highly focused laser beams which lead to material removal and redistribution on the target surface. PLA enables micro patterning of materials, cleaning of surfaces from contaminants layers and particulates and is employed in a wide range of applications in biotechnology and medicine [7]. On top of this, PLA improves bond repeatability, as well as waste and cost reduction, and is also prone to automation. Previous literature works have indicated that PLA promotes significant modifications of surface morphology [8,9] and chemistry [10, 11], leading to improvement in surface wetting $[12,13]$ and mechanical interlocking $[12,14]$, with consequent increases in bond toughness $[15,16]$ and long term joint stability [18]. It may also outperform traditional joining techniques, such as riveting and fusion welding when dealing with dissimilar materials [19].

The objective of the present work is to survey the effect of Yb-fiber (1064 nm) PLA as surface pre-treatment of copper substrates for adhesive bonding. Nanosecond pulsed $\mathrm{Yb}$ lasers are provided with high vibrational stability and extended lifetime, and are often used to machine small to large scale metal surfaces in reliable fashion and at low cost. The model material system selected for the experiments is represented by copper substrates (CuZn40) bonded with an epoxy adhesive. The CuZn40 has good mechanical, thermal, corrosion and electrical properties. The good formability of the alloy has led to a vast number of applications in several industries, e.g. pipe fitting, domestic taps, radiator valves, gas appliances, window and door furniture, architectural panel sheets, large nuts and bolts, condenser plates and heat exchangers. However difficulties arise concerning joining, because traditional fusion welding is strongly affected by the presence of zinc, which evaporates during welding with a detrimental effect on the joint microstructure, which eventually features porous and weak layers of copper and copper oxide. It follows that the fabrication of high-quality and reliable joints demands 
alternative joining techniques.

Yb-fiber PLA is herein carried out in order to impart significant modifications in surface topography and chemistry. An assessment of surface characteristics, both in terms of topography and chemistry, is firstly made for a variety of laser speed. Based on the results of surface analyses, a proper combination of processing parameters is selected and adhesive bonded T-peel joints are prepared and tested. The T-peel test configuration is very advantageous when testing flexible adherents because samples can be easily fabricated and closely resembles actual bonded structures, e.g. automotive components $[3,20,21,22]$. Moreover it is very convenient to compare adhesives or surface treatments and delivers useful insights about the ability of the joint to resist debonding. The global load-displacement response of laser treated samples is herein compared with those obtained from baseline joints made-up with sanded substrates. Meaningful metrics (e.g. peak load, total dissipated energy) are extracted from the global response in order to make quantitative assessments among the various sets of results. Post failure SEM analyses of fractured surfaces are then performed to resolve the mechanisms of failure and analyze the extent of mechanical interlocking at the adhesive/substrate interface. Finally, finite element simulations, based on the use of the cohesive zone model of fracture [16, 23, 24], are carried out to infer the enhancement of bond toughness that follows to PLA.

\section{Experimental details}

\subsection{Materials and surface treatments}

The adhesive selected for joint fabrication is a bi-component (resin+hardener) room temperature curing epoxy (Araldite 420 A/B, Huntsman, Salt Lake City, UT, USA). It is a structural adhesive with very high shear and peel strengths which bonds materials such as metals, composites and thermoplastics. The basic mechanical properties of the 
adhesive provided by the manufacturer through tensile tests are as follows: Young's modulus, $E=1.5 \mathrm{GPa}$; elongation at break $\epsilon_{f}=4.6 \%$; tensile strength, $\sigma_{f}=29 \mathrm{MPa}$. The substrates consisted of thin copper foils (CuZn40) with nominal thickness equal to $t=0.5 \mathrm{~mm}$. CuZn40 copper alloy usually contains $59 \% \div 63 \%$ in weight of $\mathrm{Cu}, \mathrm{Pb}<0.3 \%$, $\mathrm{Fe}<0.3 \%$ and $\mathrm{Zn}$ (balance). It is this high quantity of zinc in brass which leads to a dual phase (duplex) structure, i.e. alpha-beta brasses. Notice that alpha-beta brasses have higher hardness, strength, and in general better mechanical properties. Also brasses of higher zinc contents have a lower cost and thus influence the total life-time costs. The mechanical properties of the alloy were determined through dedicated tensile tests and are given as follows: Young's modulus $E=90 \mathrm{GPa}$; elongation at break $\epsilon_{f}=12.5 \%$; yielding stress $\sigma_{y}=85 \mathrm{MPa}$.

Copper substrates have been surface treated using a $1.06 \mu \mathrm{m}$ ytterbium fiber laser (PLS6MW Multi-wavelength Laser Platform by Universal Laser Systems, NY, USA) with a $30 \mathrm{~W}$ maximum output average power. In PLA a strongly localized area of the target is heated by the laser pulses, while the surrounding is basically unaffected. The spatial and temporal localizations of laser-material interaction give raise to very large heating/cooling rates and small material volumes are subjected to the thermal induced defects [7]. Surface modifications are generally imparted by controlling adjustable laser process parameters, such as laser average power, laser scanning speed (i.e. speed of the beam relative to the substrate) and spacing (i.e. laser pitch). Moreover, actual depth and quantity of affected material mainly depend on the energy density, or pulse fluence $\left(F_{p}\right)$, which is transmitted to the target surface:

$$
F_{p}=I_{p} \cdot t_{p}=\frac{W_{a v e}}{f \cdot A_{s}}
$$

where $I_{p}$ represents the laser irradiance, $t_{p}$ is the laser pulse duration, $W_{\text {ave }}$ is the average output power, $f$ the pulse frequency and $A_{s}$ is the spot size. Preliminary analyses have shown that laser power significantly affects the resulting morphological modifications. 
Indeed, for a given pulse frequency, an average power below $30 \mathrm{~W}$ could not induce any significant variation in surface morphology. In addition, it was observed that a laser spacing equal to $30 \mu \mathrm{m}$ allowed the whole target surface to receive laser processing and to be fully treated. For these reasons the subsequent processing was executed at 30 $\mathrm{W}$ and $30 \mathrm{kHz}$ and pattern density was held constant by holding the pitch fixed to 30 $\mu \mathrm{m}$. Therefore surface processing was always carried out at the maximum pulse fluence allowed by the available in-lab hardware $\left(F_{p}=200 \mathrm{~J} / \mathrm{cm}^{2}\right)$, while the laser scanning speed was varied in the range $(50 \div 500) \mathrm{mm} / \mathrm{s}$. Notice, finally, that compressed air was injected onto the work surface to suppress potential combustion flame and blow away smoke and prevent debris accumulation during the process. The use of compressed air in the working area can provide superior processing results and prevent melt-back on the lens. A set of substrates was processed replacing compressed air with nitrogen and the associated potential implications in terms of oxidation and joint performance were also surveyed. A summary of the processing parameters considered for the experimental campaign is given in Tab. 1.

\subsection{Surface morphology and composition}

A Scanning Electron Microscope (FEI Quanta 200) was used to analyze surface morphological modifications. In addition, backscattered electron analysis was also carried out to probe the fractured surfaces and highlight mechanical keying of the adhesive within the substrates. Surface chemistry was monitored through X-ray Photoelectron Spectroscopy (XPS) carried out using a Kratos AXIS Ultra DLD spectrometer (KRATOS Analytical, Wharfside, UK) equipped with a monochromatic Al-K $\alpha$ anode emitting X-ray source of photon energy $\mathrm{h} \nu=1486.6 \mathrm{eV}$. Measurements were performed at power of radiation beam equal to $150 \mathrm{~W}$ and the system was provided with a multichannel plate and delay line detector under a vacuum $\left(10^{-9} \mathrm{mbar}\right)$. Measurements were

performed in hybrid mode using electrostatic and magnetic lenses. All spectra were 
recorded using an aperture slot of $300 \mu \mathrm{m} \times 700 \mu \mathrm{m}$. The survey and high-resolution spectra were collected at fixed analyzer pass energies of $160 \mathrm{eV}$ and $20 \mathrm{eV}$, respectively. Samples were mounted in floating mode in order to avoid differential charging. Charge neutralization was required for all samples. Binding energies were referenced to the $\mathrm{C}$ 1s peak of $(\mathrm{C}-\mathrm{C}, \mathrm{C}-\mathrm{H})$ bond which was set at $285.0 \mathrm{eV}$. Measurements were made at an angle of photoelectron emission of $0^{\circ}$, which corresponds to an average $10 \mathrm{~nm}$ examined thickness.

\subsection{Fabrication of CuZn40/epoxy joints}

Mechanical testing of the joint was carried out using the T-peel test coupon. The T-peel test is an adhesion test involving large adherents deflections. Adherents are relatively flexible, bonded together and loaded in peel. The geometry has been standardized by ISO, ASTM and BS [3]. Since it involves the propagation of a crack it is also considered to be a fracture test.

For sample fabrication, CuZn40 plates $\left(100 \times 100 \mathrm{~mm}^{2}\right)$ were laser ablated over a $60 \times 100 \mathrm{~mm}^{2}$ area; grit and debris formed during the processing were cleaned from the surface by exposing it to a compressed air flow. After surface pretreatment the plates were cut down into $15 \times 100 \mathrm{~mm}^{2}$ substrates, degreased with acetone and subsequently bonded using the epoxy adhesive. The adhesive layer thickness was controlled by using $250 \mu \mathrm{m}$ thick optical fibers deployed in the longitudinal direction (i.e. x-direction in Fig.1). Weights were placed to hold the substrates in place and squeeze out any excess of adhesive. After curing at room temperature for 24 hours, the samples were gently clamped along the bonded area and the un-bonded substrates were bent by wedge splitting to confer the desired T-shape to the joint. To facilitate bending, a release film (A6000 Release Film, Richmond Aerovac, USA) was deployed over $40 \mathrm{~mm}$ of the total overlap length $(L=100 \mathrm{~mm}$ ). This procedure was deemed suitable for the current study because the use of $0.5 \mathrm{~mm}$ thick copper substrates did not require strong gripping and/or 
wedge forces to enable bending. As a result, optical microscopy analyses indicated that there was no damage induced in the bond-line after fabrication. Special care was taken in order to ensure a proper alignment of the bent portions, which were then coupled with the grips of an electro-mechanical tensile testing machine. The overall dimensions of the fabricated T-peel joints are given in Fig. 1(a). Since substrates warping and/or distortions occasionally led to a variable adhesive layer thickness, all samples were probed through optical microscopy and those showing substantial deviations from the nominal thickness $(\geq 10 \%)$ were not employed in mechanical tests. Finally, in order to analyze the subsequent improvements associated to PLA, additional samples were fabricated using sanding as surface pre-treatment. To this aim 400-grit sandpaper (3M Wetordry ${ }^{\mathrm{TM}}$ ) was employed.

\subsection{Mechanical testing of T-peel joints}

Mechanical tensile tests were carried out using an electro-mechanical tensile testing machine (Instron 5944, Norwood, Massachusetts, USA) equipped with a $2 \mathrm{kN}$ load cell; the cross-head feed rate was set equal to $5 \mathrm{~mm} / \mathrm{min}$, while a slower initial displacement rate was used to pre-crack the samples. Notice that the ablation pattern lines were orthogonal to the direction of crack propagation since laser scanning was made in the z-direction (cfr. Fig. 1(a)).

The deformed configuration assumed by the sample during mechanical testing affects the results. In particular, if the fracture process zone has constant size, and no stickslip occurs, the distance from the line of action of the applied load to the crack front $(d)$ does not evolve during crack propagation. It follows that self-similar (or steady state) debonding occurs. On the other hand, if self-similar debonding is not achieved, the previous distance continues to increase as the crack growths [3]. The deformed configurations are shown in the schematic reported in Fig. 1(b) and (c). The T-peel strength is given as the average force after the peak $\left(P_{\text {ave }}\right)$ needed to debond the joint 
divided by the sample width (cfr. Fig. 1(b)). The apparent peel energy is twice the Tpeel strength [3]. Notice that the apparent peel energy matches the fracture toughness of the joint provided the failure process does not involve plastic dissipation in the adherents. If plastic deformations of the metal substrates are involved, the apparent peel energy embeds the plastic work carried out to bend the substrates and to partially straighten the debonded adherents, which in some cases could be one or two order of magnitude higher than the fracture energy of the adhesive. In such cases, advanced modeling techniques could be employed to segregate plasticity from total dissipation and obtain the bond toughness $[14,15,16,17]$. Additional data that can be extracted from a T-peel test are the peak force $\left(P_{\text {peak }}\right)$, the total dissipated energy (area under the load-extension curve) or the total energy per unit debonded area. Selected metrics, from those mentioned above, are employed herein to describe the outcome of the experimental measurements.

\section{Results and discussion}

\subsection{Analysis of surface morphology}

As produced and sanded surfaces are reported for comparison in Fig. 2. The surface of as produced copper substrates is basically featureless, see Fig. 2(a), however after sanding random ridges and grooves were produced, as shown in Figs. 2(b-d), which can promote an increase in roughness and surface area available for bonding.

Using laser irradiation a fraction of the laser beam energy is absorbed by the material, thus promoting material melting and/or vaporization which ultimately lead to modifications in surface morphology and chemistry. A comparison among the SEM pictures reported in Fig. 3 demonstrates the effect of PLA, which was carried out at maximum fluence and for varying speed levels. The laser speed had an important effect on the resulting morphology, i.e. the degree of modifications substantially increased as the laser speed decreased, see Fig. 3. 
Moreover, the presence of recast material, a multitude of sharp asperities and metallic particles can be also observed. Spherical particles are likely to be originated by the rapid condensation of the expanding gas or plasma during cooling $[7,8]$. The so obtained morphological modifications can increase contact surface and enhance mechanical interlocking since the generated cavities and asperities may be able to retain the adhesive and resist debonding. However, intricate interfacial morphologies might be detrimental for surface wetting, because in conjunction with capillarity forces and adhesive viscosity can adversely affect gap filling capabilities, especially when using cold curing adhesives [14].

High resolution SEM images reported in Figs. 3(c), (f) and (i) indicated substantial differences in the surface features displayed by the treated substrates. Apparently, a structured oxide layer was formed that seems to have sub-micron scale features. The layer occasionally shows cracks and delamination as shown in Figs. 3(b) and (e). For this reason, oxide adhesion to the bulk alloy may be lower than its adhesion to the adhesive thereby representing a critical factor able to trigger interfacial failure. Such an issue was already observed on laser textured titanium surfaces [9].

\subsection{XPS measurements}

Modification of surface chemistry was analyzed through XPS analyses. Figure 4 shows the survey spectrum for Cu2p. The largest peak at the right end side is given by the superposition of three curves corresponding to different compounds. Curve fitting provided the following information. The largest peak signal was identified to be metallic copper, although the peak is also compatible with cuprous oxide, $\mathrm{Cu}_{2} \mathrm{O}$; indeed, as noted in [27], the two compounds have very similar binding energies and are difficult to distinguish in $\mathrm{Cu} 2 \mathrm{p}$ spectrum. Moreover, cupric oxide, $\mathrm{CuO}$, and copper hydroxide,

$\mathrm{Cu}(\mathrm{OH})_{2}$, were also identified. From this standpoint, the appearance of some of the satellite peak signals, which are shifted from the large peak signal, corresponds to $\mathrm{Cu}^{2+}$ 
and suggests the presence of $\mathrm{CuO}$. The results also show that peak intensity for metallic copper increases with the laser speed, which implies that a higher degree of laser ablation leads to removal of metallic $\mathrm{Cu}$ (and/or $\mathrm{Cu}_{2} \mathrm{O}$ ). Notice that the spectra pertaining to samples processed in air and nitrogen do not differ significantly. As a result we do not expect the nitrogen gas to play a major role on the surface chemistry and mechanical response of the joints.

In order to investigate surface oxidation, the spectra O1s pertaining to the different processing conditions have been analyzed. The analysis of survey spectra, which are reported in Fig. 5, confirms the presence of $\mathrm{CuO}$. In particular, there are two peaks, the one on the right hand side corresponds to $\mathrm{CuO}$ while the one on the left pertains to $\mathrm{Cu}(\mathrm{OH})_{2}$. As a result of PLA the relative intensity of the two peaks is modified. When processing is carried out at the highest speed, independently of the kind of processing gas, both peaks are reduced and any of them prevails. However, as the speed is decreased, the $\mathrm{Cu}(\mathrm{OH})_{2}$ peak is reduced and that pertaining to $\mathrm{CuO}$ prevails. This might be explained considering that the local temperature increases as the laser speed decreases, and that the formation of $\mathrm{CuO}$ is sustained at high temperature $\left(400^{\circ} \mathrm{C} \div 600^{\circ} \mathrm{C}\right)$ [28]. This is in agreement with the SEM observations, which display an evolution of the sub-micron scale oxide morphology as the speed increased. It is worth noting that the formation of $\mathrm{CuO}$ has been shown to have a beneficial effect on the strength of adhesive joints. Indeed, it improves wettability and, in turn, increases the degree of interfacial contact with the adhesive enabling mechanical interlocking [28]. Figure 6 shows copper, oxygen, nitrogen and carbon abundance (atomic percentage) as a function of the laser speed. The oxygen abundance increases after laser processing and the overall variation with laser speed is not significant. On the other hand, the abundance of copper shows an overall decreases with laser speed. Carbon abundance before and after processing was also recorded. However, carbon content is influenced by several impurities, for instance it might be related to the presence of dust from atmospheric contamination [25]. 


\subsection{Mechanical tests}

Considering the results of surface morphology and chemistry combined, and that laser processing at $50 \mathrm{~mm} / \mathrm{s}$ caused the occurrence of significant thermal distortions of the substrates, subsequent sample processing for mechanical tests was carried out by considering $\mathrm{V}=250 \mathrm{~mm} / \mathrm{s}$ and $500 \mathrm{~mm} / \mathrm{s}$. In summary, five batches of T-peel joints were prepared, one batch was fabricated by using sanding as surface preparation, and four batches using PLA. The latter were obtained by combining $250 \mathrm{~mm} / \mathrm{s}$ and $500 \mathrm{~mm} / \mathrm{s}$ laser scanning speed and the use of either air or nitrogen as processing gas.

Typical load displacement responses of laser treated $(250 \mathrm{~mm} / \mathrm{s}$ in air $)$ and sanded samples are reported in Fig. 7. The response curve from the peel test consists of an initiation stage which is followed by a steady state peeling regime where the peeling force attains a constant value. Since self-similar and stable debonding was achieved, the distance from the line of action of the applied load to the crack front $(d)$ was constant during debonding (cfr. Fig. 1). In such cases the crack tip propagates at a rate corresponding to half of the moving cross-head. Therefore during debonding the load acting on the moving cross-head moves twice the distance [7]. Failure was always self-similar (steady state) in our tests and a small degree of asymmetry in the deformed configuration was observed on few samples. This feature was attributed to the crack path, which was actually closer to one of the interfaces. Visual inspection of fractured surfaces suggested that PLA enabled a transition from adhesive (apparent interfacial fracture) to cohesive failure for the T-peel joints.

Comparing the global responses it is clear that PLA promoted a significant increase in the apparent peel energy. Specifically, joint deformation in the course of fracture was strongly affected by the level of adhesion. Indeed, weaker adhesion required smaller forces to debond the sample and involves limited plasticity. However, as the adhesion

increases, the larger forces and bending moments induced plastic bending and enabled the energy dissipation potential associated with substrates yielding. 
In order to draw quantitative conclusions a summary of the whole set of experimental results is given by the bar diagrams reported in Fig. 8. The diagram displays and compares the peak force and the average force after the peak, the T-peel strength and the total absorbed energy per unit bonding area. The latter is the total area under the load-displacement curve and represents the absorbed energy during the process. Notice that the quoted values have been normalized with respect to samples treated at 250 $\mathrm{mm} / \mathrm{s}$ in air, that provided the best response in mechanical tests (i.e. $P_{\text {peak }}=143 \mathrm{~N}$, $P_{\text {ave }}=87 \mathrm{~N}$, T-peel strength $=5.8 \mathrm{~N} / \mathrm{mm}$, total energy per bonded area $=5.7 \mathrm{~kJ} / \mathrm{m}^{2}$ ). All quantities show major increases after laser ablation. The results also show that laser processing using nitrogen as service gas did not bring any significant difference with respect to samples treated in air.

Therefore, the improvement in adhesive bonding after PLA is attributed to the increase in surface area, the generation of surface features, also of sub-micron scale, which improved wetting and acted as locking sites enabling mechanical interlocking $[8,9,12,14]$. It has been observed in previous related work that all these effects combined may also lead to the formation of a composite interfacial region (i.e. adhesive interlocked within substrates asperities) able to enhance joint strength [10].

\subsection{Analysis of damage mechanisms within the adhesive bond line}

As discussed in the previous section, visual inspection of fractured surfaces testified that PLA enabled the occurrence of cohesive fracture within the adhesive layer. A detailed SEM analysis can be an extremely useful tool in order to precisely assess the damage mechanisms that led to bondline failure. SEM pictures reported in Fig. 9 show the appearance of fracture surfaces taken from the mating substrates which were treated at $30 \mathrm{~W}$ and $250 \mathrm{~mm} / \mathrm{s}$. Notice that cohesive failure was observed for laser treated samples, while sanded ones, not shown here for conciseness, failed in apparent interfacial fashion. 
Figure 9(a) displays the adhesive interlocked within the cavity created by the ablation process and the magnified view given in Fig. 9(b) shows that occasionally the adhesive was cleaved from the patterns resulting in apparent interfacial decohesion. However, Figure 9(c), that is the back scattered version of Fig. 9(b), demonstrates that despite the apparent interfacial decohesion, the adhesive (dark regions) was actually able to interlock with the microscopic surface features generated by PLA. Figure 9(d) shows the fracture surfaces from the opposite substrates and demonstrates that more adhesive was retained by one of the substrates, while the magnification given in Fig. 9(e) shows an highly strained region where cohesive failure occurred. The corresponding back scattered version, Fig. 9(f), highlights that portions of copper oxide were detached from the substrate. Therefore, the adhesive was able to interlock within surface asperities, however the oxide layer was also detached from the mating surface. This feature is further illustrated in Fig. 9(g) where a typical patch of oxide layer is reported. It is possible to note from Figs. 9(h) and (i) that surface morphology of the detached oxide layer closely resembles that observed in the SEM pictures reported earlier in the paper. Notice that failure of the laser generated oxide layer was also observed in titanium alloys [9].

\subsection{Segregating the bond toughness from the apparent peel energy}

The apparent peel energy determined in mechanical tests includes substrates plastic dissipation and, as a result, the actual bond toughness of the joint should be considerably lower. In order to segregate the bond toughness from the apparent peel energy, finite element simulations based on the use of the cohesive zone model (CZM) of fracture were carried out. The CZM lends itself to the analysis of plastically deforming adhesive joints because it allows to make a partition between the energy required to advance the crack and that required by the plastic deformation of the bonded substrates [16, 17, 23, 24]. In the CZM approach, material failure is characterized by a traction-separation relation which links the cohesive traction and the relative displacement across cohesive surfaces. 
The peak stress and the area enclosed by the traction-separation relation are referred to as the cohesive strength and cohesive energy, respectively.

The debonding process was herein analyzed through a finite-element model of the sample. The adherends were modeled by using four nodes continuum elements available in ABAQUS/Standard (CPE4) and assuming plain strain and large displacements conditions. The uniaxial stress-strain data obtained through dedicated tensile tests was given as input in tabular form. The tensile behavior was then generalized to multiaxial stress states assuming isotropic hardening and using the von Mises yield surface. The adhesive layer was replaced by a single row of four-noded cohesive elements with finite thickness equal to the bondline thickness. It is then assumed that the role of the adhesive is to provide a traction-separation relation across the interface between the substrates $[26]$.

The stiffness matrix and the load vector of the cohesive elements are assembled in a user defined subroutine within the commercial code ABAQUS/Standard. The cohesive model employed herein to mimic the response of the adhesive is schematically shown in the insert of Fig. 10. The evolution of cohesive interaction depends on a few parameters, which include the cohesive strength, $\sigma_{\max }$, the shape parameters, $\lambda_{1}$ and $\lambda_{2}$, which allow to control the initial slope (i.e. the stiffness), the extension and position of the plateau, and the final opening width, $\delta_{f}$, after which the material is no longer able to sustain any load. Finally, the area under the traction-separation relation, $\Phi$, is the cohesive fracture energy or the bond toughness. Although there are four independent cohesive parameters that fully define the cohesive interaction $\left(\phi_{n}, \sigma_{\max }, \lambda_{1}, \lambda_{2}\right)$, previous works by the authors have shown that the simulated global load-extension curve in T-peel tests is mostly sensitive to the cohesive energy and cohesive stress, and that basic curve fitting in the plateau region allows to assess in a fairly reliable fashion the cohesive energy $[15,16]$. A similar procedure has been employed in the present work.

A comparison among experiments $(\mathrm{V}=250 \mathrm{~mm} / \mathrm{s}$, processing gas: air $)$ and simula- 
tions in the plateau region is given in Fig. 10. Notice that $\lambda_{1}$ and $\lambda_{2}$ were set equal to 0.01 and 0.5 for all simulations, while cohesive strength, which was estimated from tensile tests carried out by the manufacturer, was set equal to $29 \mathrm{MPa}$. Therefore iterations were carried out to finely tune cohesive energy and the input value that allowed to fit the experiments lies in the range $(2.07 \pm 0.63) \mathrm{kJ} / \mathrm{m}^{2}$. Using a similar procedure, the bond toughness of the remaining sets of samples was extracted and the results are summarized in Fig. 11. It is shown that the highest bond toughness was recorded for the interfaces treated in air at $250 \mathrm{~mm} / \mathrm{s}$, and that there was a jump in toughness whenever laser irradiation was used to replace sanding. Cohesive failure was observed in all laser treated samples, therefore cohesive strength was kept constant for all simulations. It is believed that the estimated value is fairly reliable for the present material system and thickness of the adhesive layer. Indeed, finite element simulations indicated a lower sensitivity of the predicted global load-displacement curve to variations around the quoted cohesive strength. This point was already observed in a previous investigation carried out by the authors on T-peel aluminum joints bonded with epoxy adhesive [16]. The bond toughness of sanded samples was also extracted and was shown to be significantly lower and lying in the range $(0.12 \pm 0.03) \mathrm{kJ} / \mathrm{m}^{2}$. Notice that failure of sanded samples was basically interfacial, therefore an additional set of finite element simulations was carried out to assess the effect of cohesive strength. The results indicated that selecting the cohesive strength in the range $(5 \div 30) \mathrm{MPa}$ had an even weaker effect on the global response. It should be noted that, since a flat substrate/adhesive interface is assumed, the modeling approach undertaken herein neglects the microscopic morphological details of laser irradiated surfaces; moreover, all the dissipation occurring in the adhesive layer (i.e. bulk plasticity) is lumped in the cohesive zone model and is included in the cohesive energy (i.e. bond toughness). Nevertheless, finite element simulations and experiments display a fairly good agreement with the force plateau region. 


\section{Conclusions and future works}

The present work reported about pulsed laser ablation of copper substrates for adhesive bonding. The results of SEM and XPS analyses combined have shown that PLA caused significant modifications of surface topography with simultaneous modification of surface chemistry. The extent of the modifications depended on laser speed - for given power and pulse frequency (i.e. for a given pulse fluence). A sub-micron structured oxide layer, with nanometer scale surface features, was observed. It was speculated that the oxide layer increased the effective contact area between the liquid adhesive and the substrate with consequent increased wettability.

T-peel tests, carried out on copper/epoxy joints, confirmed the outcome of the analyses of surface morphology and chemistry and indicated a major improvement in the apparent peel energy. The improvement was enabled by the synergistic effect arising from the increase of surface area and enhanced mechanical interlocking. However, the oxide layer also displayed cracks and delamination which eventually were detached in the course of debonding thereby testifying some degree of apparent interfacial failure. Since PLA led to an increase in adhesion, larger forces (and bending moments) were needed to severe the joints and extensive plastic dissipation was induced in the course of failure. Therefore, contrary to sanding treatment, PLA enabled to access the dissipation potential associated to adherent yielding.

For this reason, the measured apparent peel energy included the plastic work needed to induce plastic deformation in the adherents. In order to segregate the bond toughness from the apparent peel energy, finite element simulations based on the use of the CZM were carried out. Cohesive energy was finely tuned against experimental global response until a reasonable match between experiments and simulations in the plateau region was achieved. The outcome of finite element simulations indicated a substantial increase of bond toughness with respect to sanded samples. However, it is recognized 
that the modeling approach undertaken herein neglected the microscopic morphology of the interface and employed a single row of cohesive elements to mimic the bond-line. Therefore the details of the failure process occurring around the interface were spatially homogenized and the dissipation within the adhesive layer was lumped in the cohesive model. This approach can provide useful insights about the failure process and help to elucidate the increase in bond toughness, though care should be paid in using the obtained parameters to simulate the same interface under different loading conditions or substrate thickness.

Further refinements of the process are potentially possible. For instance, by manipulating processing parameters, such as laser pitch, geometry of the area treated, number of passes, specific surface textures or patterns can be generated that may have a leveraging effect on the apparent peel energy and ultimately increases the work of separation. This is the subject of our ongoing work.

\section{Acknowledgements}

The authors wish to thank King Abdullah University of Science and Technology (KAUST) for supporting this research.

\section{References}

[1] R. D. Adams, J. Comyn and W. C. Wake. Structural Adhesives Joints in Engineering, Chapman and Hall, 1997.

[2] A. J. Kinloch. Adhesion and Adhesives, Science and Technology, Chapman and Hall, 1987.

[3] L. F.M. da Silva, D. A. Dillard (Editor), B. Blackman, R. D. Adams. Testing Adhesive Joints: Best Practices, Wiley, 2012. 
[4] A. Spaggiari, E. Dragoni. Effect of mechanical surface treatment on the static strength of adhesive lap joints. J Adhes 2013; 89: p.677-696.

[5] G. W. Critchlow, D. M. Brewis. Review of surface pretreatments for aluminium alloys. Int $J$ Adhes Adhes 1996; 16: p.255-274.

[6] Directive 2008/98/EC of the European Parliament and of the council of November 9th 2008, articles 17 to 20 .

[7] C. R. Phipps. Laser ablation and its applications. Springer, 2007.

[8] M. Alfano, G. Ambrogio, F. Crea, L. Filice, F. Furgiuele. Influence of laser surface modification on bonding strength of Al/Mg adhesive joints. J Adh Sci Technol 2011; 25(11): p.1261-1276.

[9] P. Maressa, L. Anodio, A. Bernasconi, A. Gökhan Demir, B. Previtali. Effect of Surface Texture on the Adhesion Performance of Laser Treated Ti6Al4V Alloy. $J$ Adhes 2015; 91: p.518-537.

[10] E.G. Baburaj, D. Starikov, J. Evans, G. A. Shafeev, A. Bensaoula. Enhancement of adhesive joint strength by laser surface modification. Int J Adhes Adhes 2007; 27(4): p.268-276.

[11] F. L. Palmieri, K. A. Watson, G. Morales, T. Williams, R. Hicks, C. J. Wohl, J. W. Hopkins, and J. W. Connell. Laser Ablative Surface Treatment for Enhanced Bonding of Ti-6Al-4V Alloy. ACS Materials and Interfaces 2013; 5(4): p.1254-1261.

[12] M. Alfano, S. Pini, G. Chiodo, M. Barberio, A. Pirondi, F. Furgiuele, R. Groppetti. Surface patterning of metal substrates through low power laser ablation for enhanced adhesive bonding. J Adhes 2014; 90(5-6): p.384-400. 
[13] G. Rotella, M. Alfano, T. Schiefer, I. Jansen. Enhancement of static strength and long term durability of steel/epoxy joints through a fiber laser surface pretreatment. Int J Adhes Adhes, 10.1016/j.ijadhadh.2015.08.009.

[14] M. Alfano, G. Lubineau, F. Furgiuele, G. H. Paulino. Study on the role of laser surface irradiation on damage and decohesion of $\mathrm{Al} /$ epoxy joints. Int $J$ Adhes Adhes 2012; 39: p.33-41.

[15] M. Alfano, F. Furgiuele, G. Lubineau, G. H. Paulino. Simulation of debonding in $\mathrm{Al} /$ epoxy T-peel joints using a potential based cohesive zone model. Procedia Eng 2011; 10: p.1760-1765.

[16] M. Alfano, G. Lubineau, F. Furgiuele, G. H. Paulino. On the enhancement of bond toughness for Al/epoxy T-peel joints with laser treated substrates. Int J Fract 2011; 171: p.139-150.

[17] Q. D. Yang, M. D. Thouless, S. M. Ward. Analysis of the symmetrical $90^{\circ}$-peel test with extensive plastic deformation. J Adhes 2000; 72: p.115-132.

[18] R. Rechner, I. Jansen, E. Beyer. Influence on the strength and aging resistance of aluminum joints by laser pre-treatment and surface modification. Int $J$ Adhes Adhes 2010; 30(7): p.595-601.

[19] M. Alfano, G. Ambrogio, L. Filice, F. Furgiuele, E. Gallus, D. D'Antuoni. On the Performance of Welded, Riveted and Adhesive Bonded Al/Mg Sheet Metal Joints. Key Eng Mat 2011; 473: p.237-242.

[20] D. Castagnetti, E. Dragoni, A. Spaggiari. Failure analysis of bonded T-peel joints: Efficient modelling by standard finite elements with experimental validation. Int $J$ Adhes Adhes 2010; 30: p.306-312. 
[21] L. D. R. Grant, L. F. M. da Silva, R. D. Adams. Effect of the temperature on the strength of adhesively bonded single lap and $\mathrm{T}$ joints for the automotive industry. Int $J$ Adhes Adhes 2009; 29(5): p.535-542.

[22] L. F. M. da Silva, R. D. Adams. The strength of adhesively bonded T-joints. Int J Adhes Adhes 2002; 22(4): p.311-315.

[23] Q. D. Yang, M. D. Thouless, S. M. Ward. Numerical simulations of adhesively bonded beams failing with extensive plastic deformation. J Mech Phys Solids 1999; 47: p.1337-1353.

[24] M. S. Kafkalidis, M. D. Thouless, Q. D. Yang, S. M. Ward. Deformation and fracture of an adhesive layer constrained by plastically-deforming adherends. J Adhes Sci Technol 2000; 14: p.1593-1607.

[25] R. Rechner, I. Jansen, E. Beyer. Optimization of the aluminum oxide properties for adhesive bonding by laser surface pretreatment. J Laser App 2012; 24(3): art. no. 032002 .

[26] M. Alfano, G. Lubineau, G. H. Paulino. Global sensitivity analysis in the identification of cohesive zone models using full-field kinematic data. Int J Solids Struct 2015; 55: p.66-78.

[27] J. N. Borges, T. Belmonte, J. Guillot, D. Duday, M. Moreno-Couranjou, P. Choquet, H.-N. Migeon. Functionalization of Copper Surfaces by Plasma Treatments to Improve Adhesion of Epoxy Resins. Plasma Process Polym 2009; 6: p.S490-S495.

[28] K. Cho, E. C. Cho. Effect of the microstructure of copper oxide on the adhesion behavior of epoxy/copper leadframe joints. J Adhes Sci Technol 2000; 14(11): p.1333-1353. 
Table 1: Laser processing parameters.

\begin{tabular}{lc}
\hline \hline Process parameter & Value \\
\hline Laser wavelength $[\mathrm{nm}]$ & 1064 \\
Focal distance $[\mathrm{mm}]$ & 46 \\
Spot size $[\mu \mathrm{m}]$ & 25 \\
Pulse repetition rate $[\mathrm{kHz}]$ & 30 \\
Pulse duration $(\mathrm{FWHM})[\mathrm{ns}]$ & $>10$ \\
Pulse energy (max) $[\mathrm{mJ}]$ & 2.0 \\
Max average power $[\mathrm{W}]$ & 30 \\
Laser speed $(\mathrm{V})[\mathrm{mm} / \mathrm{s}]$ & $50 / 250 / 500$ \\
Processing gas $[-]$ & air $/$ nitrogen \\
\hline
\end{tabular}

FWHM: full width at half maximum 


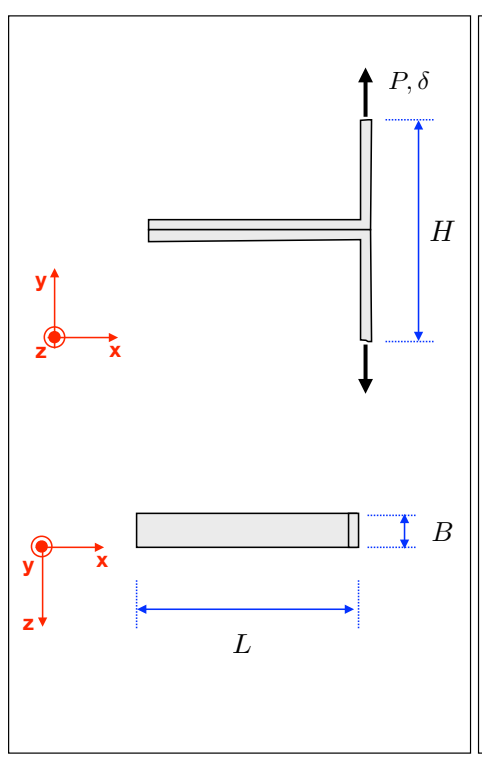

(a)

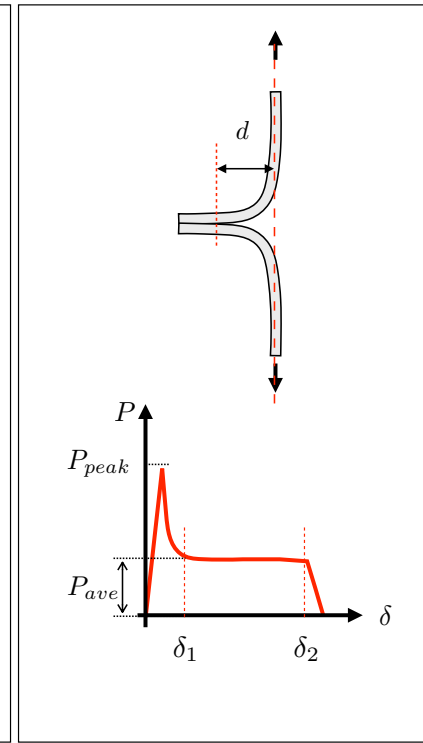

(b)

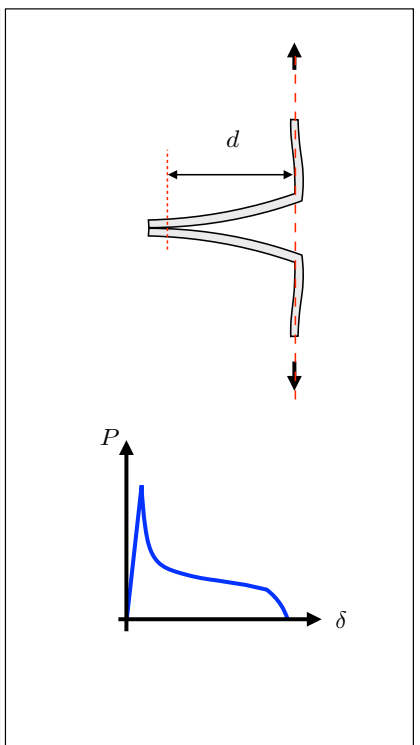

(c)

Figure 1: (a) T-peel test configuration and characteristic geometrical dimensions ( $H=80 \mathrm{~mm} ; L=60$ $\mathrm{mm} ; B=15 \mathrm{~mm}$ ); (b) deformed shape and schematic of typical load-displacement response for a T-peel sample which achieves self-similar debonding during crack growth (i.e. $d=$ const.); note that $P_{\text {peak }}$ is the peak load, while $P_{a v e}$ is the average load after the peak, i.e. the average load determined in the plateau region delimited by the opening displacements $\delta_{1}$ and $\delta_{2}$. (c) Schematic of typical T-peel sample failing without achieving self-similar (steady state) debonding. 

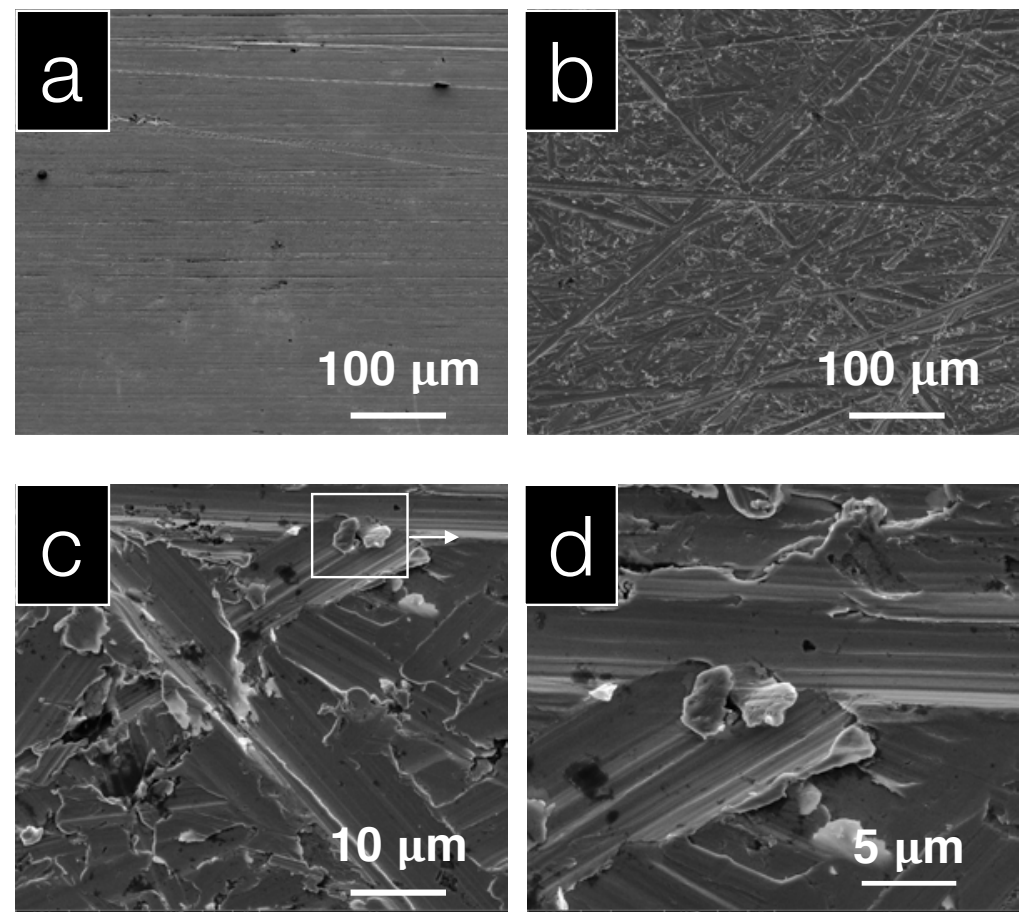

Figure 2: High resolution SEM images of (a) as received and (b to d) sanded CuZn40 substrates at different magnification levels. 

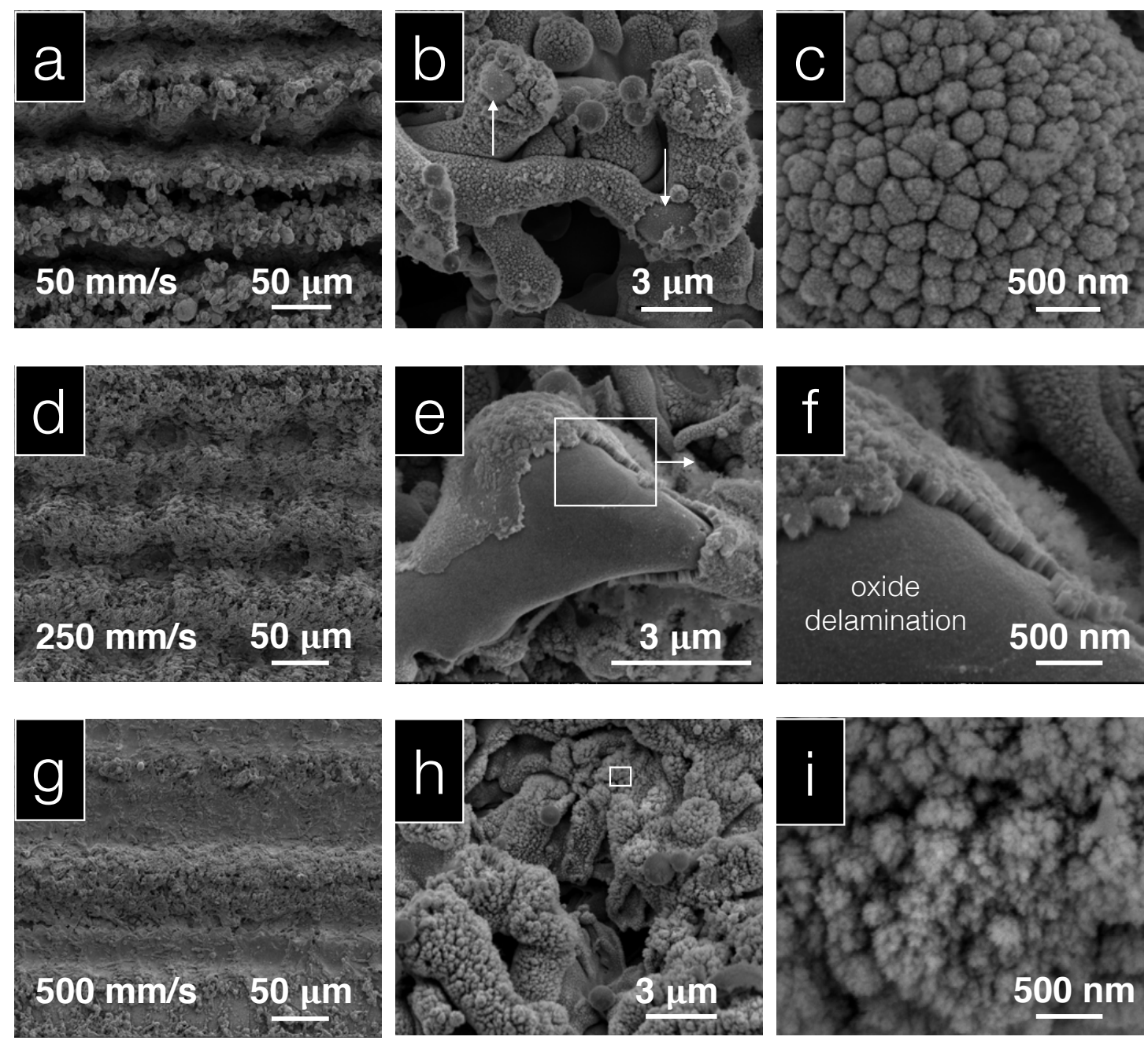

Figure 3: High resolution SEM images of laser treated CuZn40 substrates at power $\mathrm{P}=30 \mathrm{~W}$ and speed $\mathrm{V}=50 \mathrm{~mm} / \mathrm{s}$ (a to c), $250 \mathrm{~mm} / \mathrm{s}$ (d to f) and $500 \mathrm{~mm} / \mathrm{s}$ (g to i). 


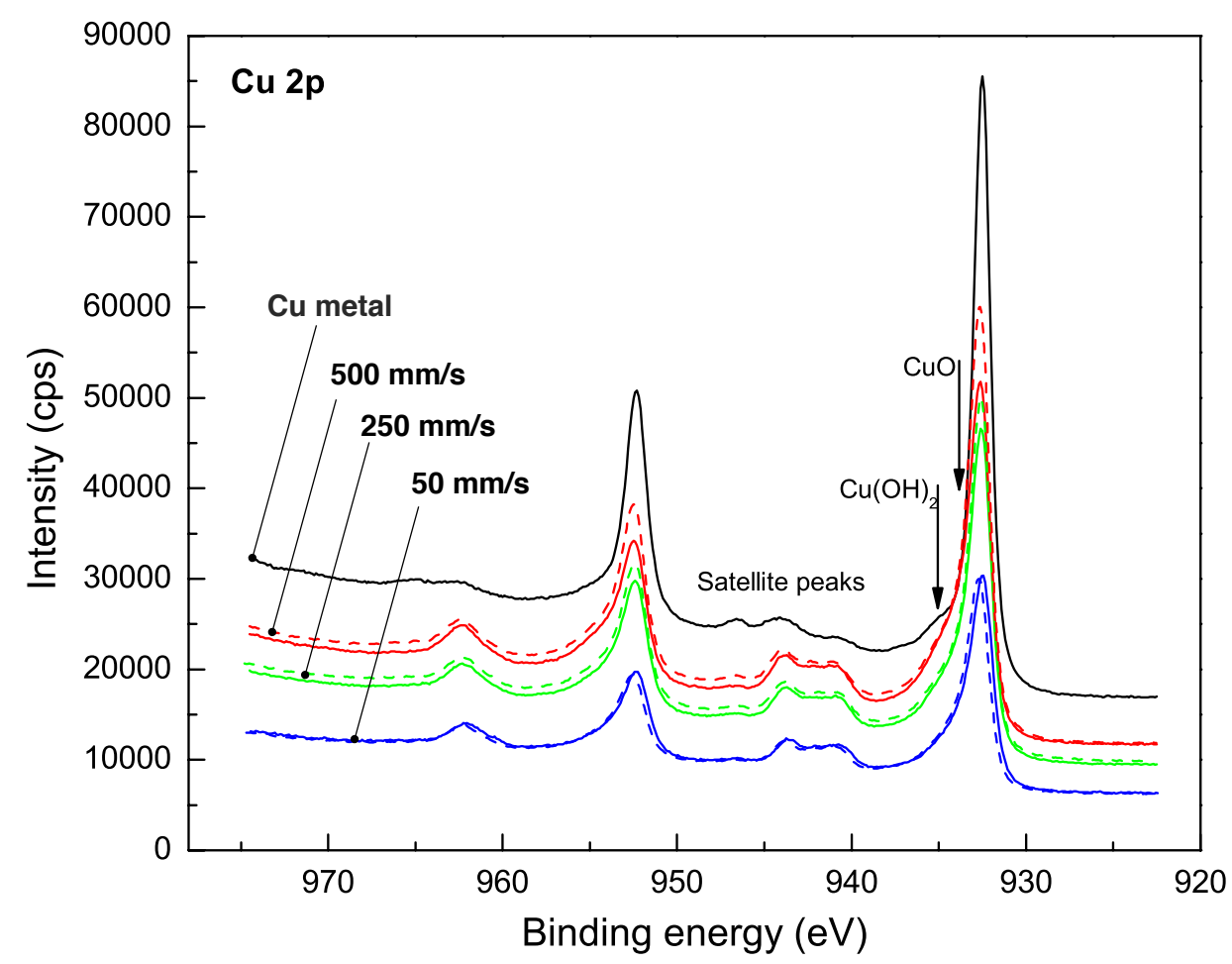

Figure 4: Cu2p XPS survey spectra of laser treated CuZn40 for various set of processing parameters. The dashed curves are referred to samples treated using nitrogen as processing gas. 


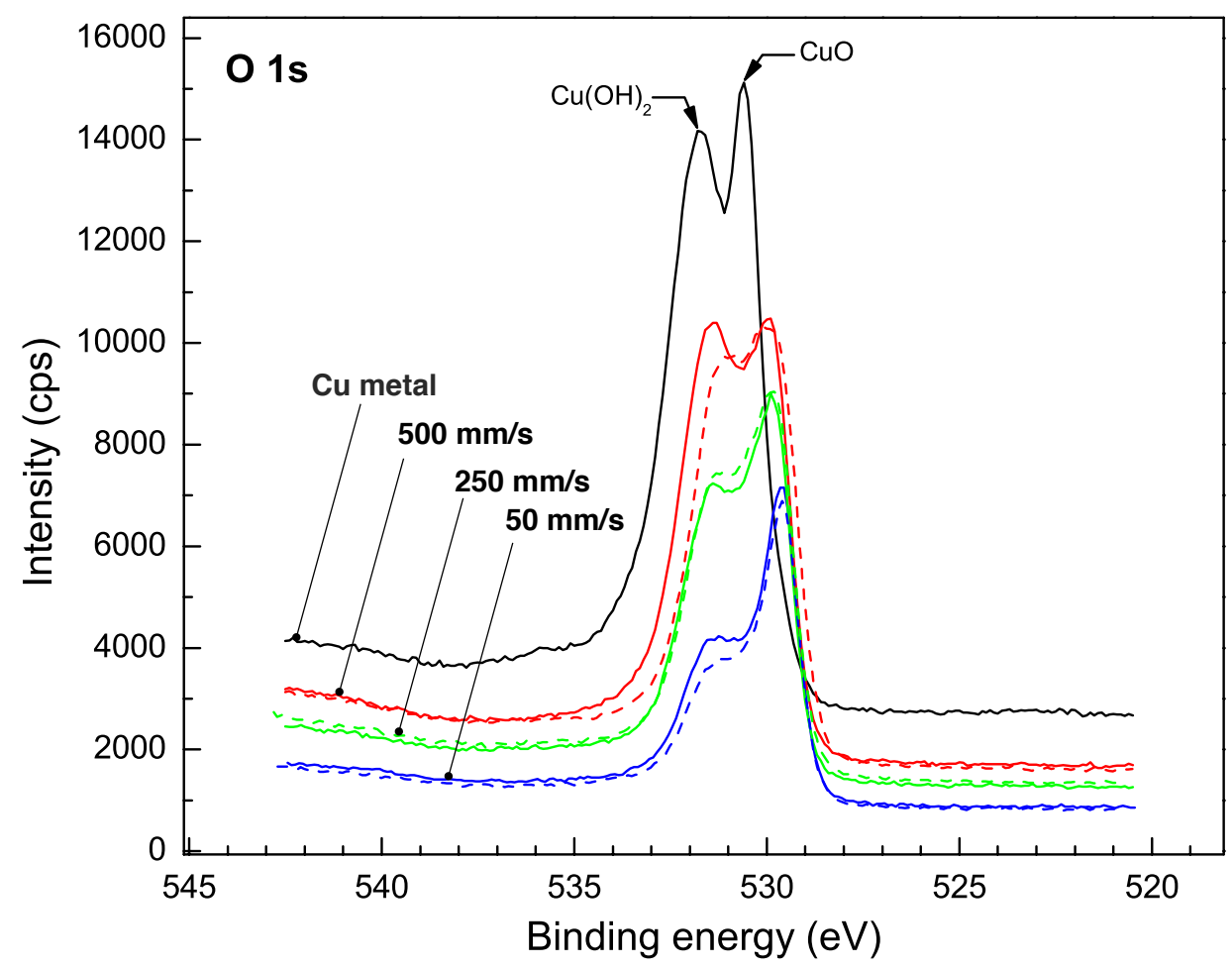

Figure 5: Expanded view of the O1s region pertaining to laser treated CuZn40 for various set of processing parameters. The dashed curves are referred to samples treated using nitrogen as processing gas. 


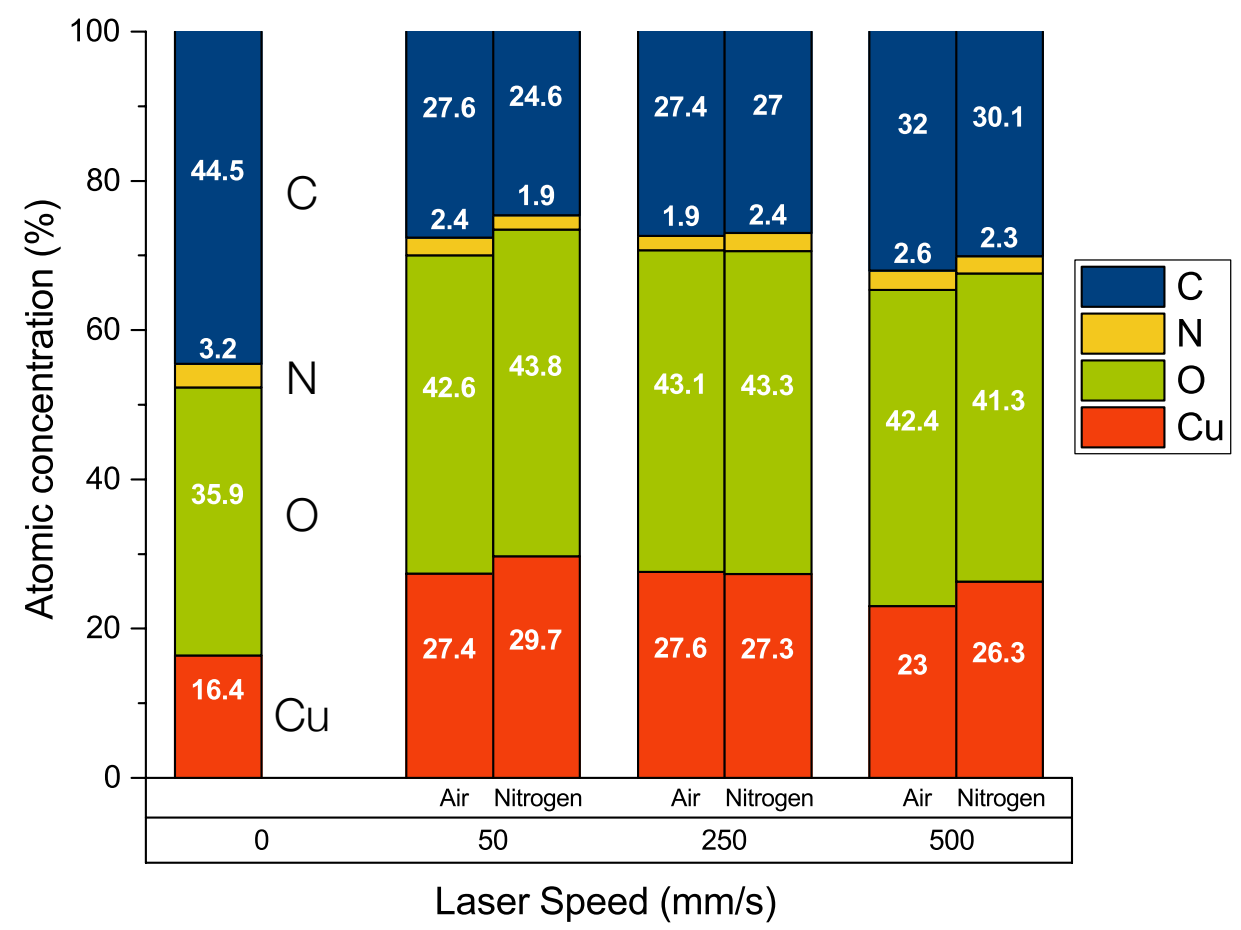

Figure 6: Atomic percent abundance of copper, oxygen, nitrogen and carbon as determined through the analysis of XPS scan survey spectra. 


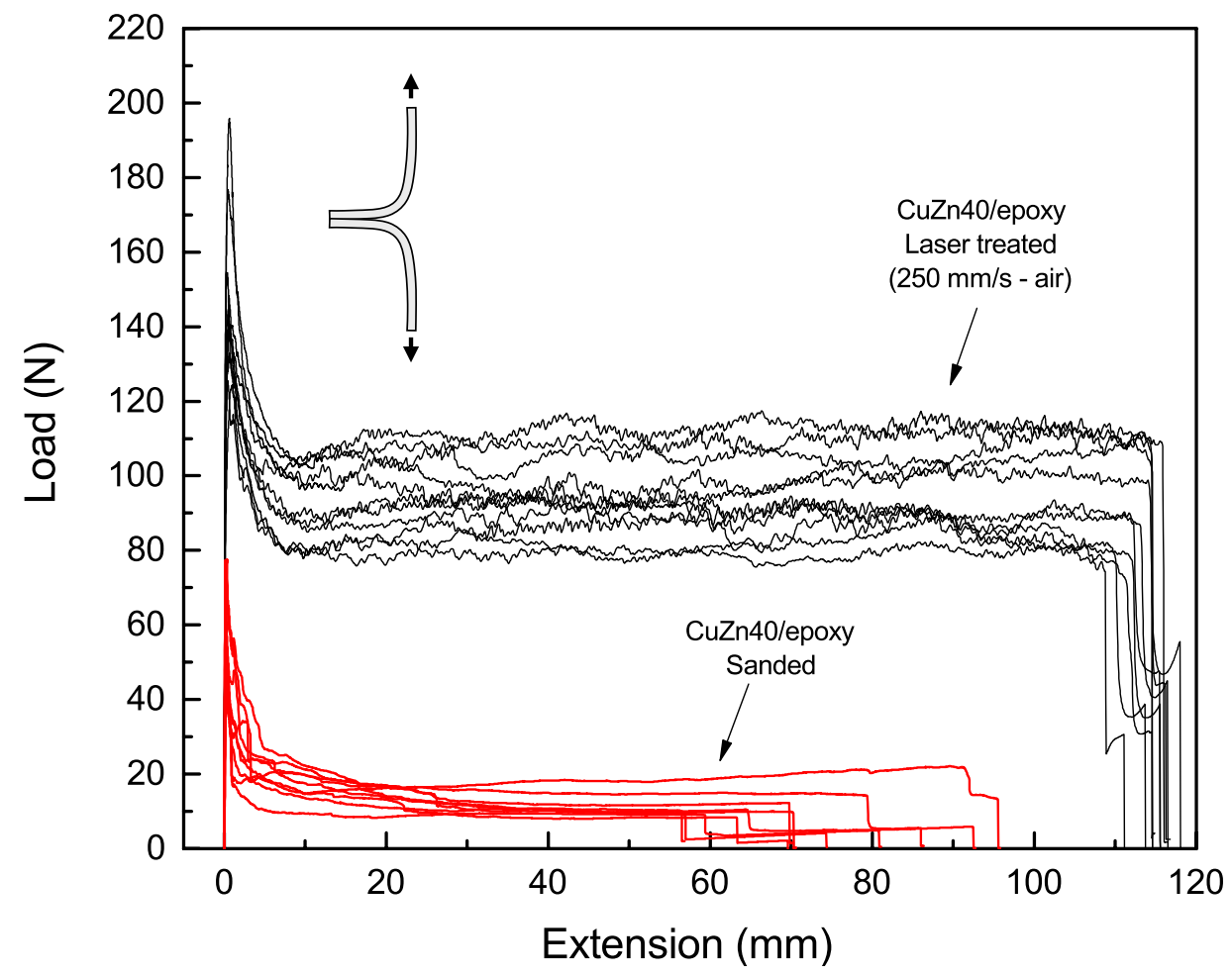

Figure 7: Comparison among the load-displacement responses of sanded and laser treated $(250 \mathrm{~mm} / \mathrm{s}$ in air) T-peel joints. Self similar debonding was achieved in both cases. 

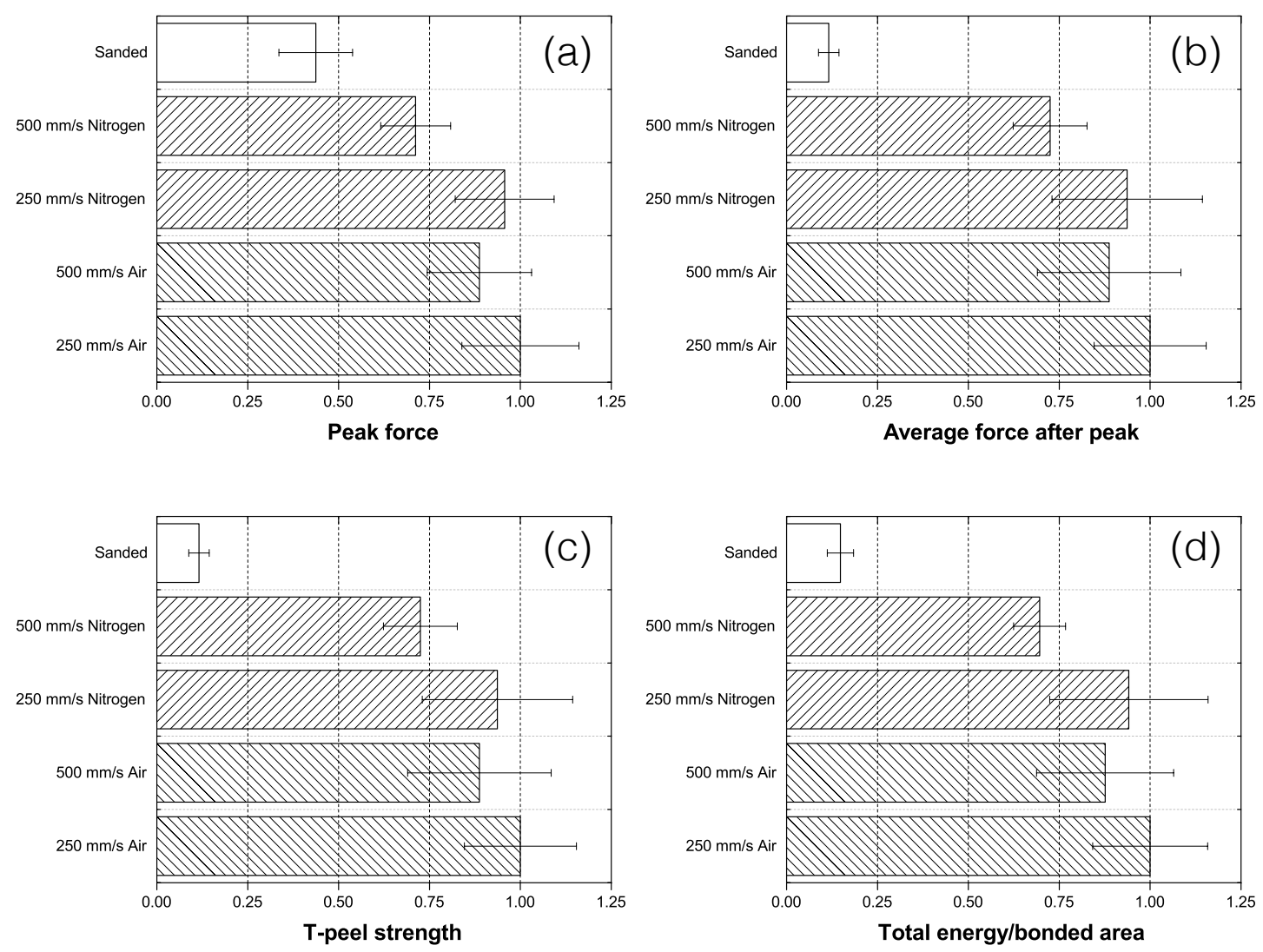

Figure 8: T-peel tests results summary. All quantities are normalized with respect to samples treated in air at $250 \mathrm{~mm} / \mathrm{s}$. (a) Peak force $\left(\mathrm{P}_{\text {peak }}\right)$ recorded during the tests; (b) average force after the peak $\left(\mathrm{P}_{\text {ave }}\right)$ achieved in the plateau region; (c) T-peel strength as defined in the ASTM D1876-08; (d) total energy absorbed (per unit bonded area) during the tests. Notice that the quoted numbers represent averaged data over a minimum of five tests. The error bar represent the corresponding standard deviation. 

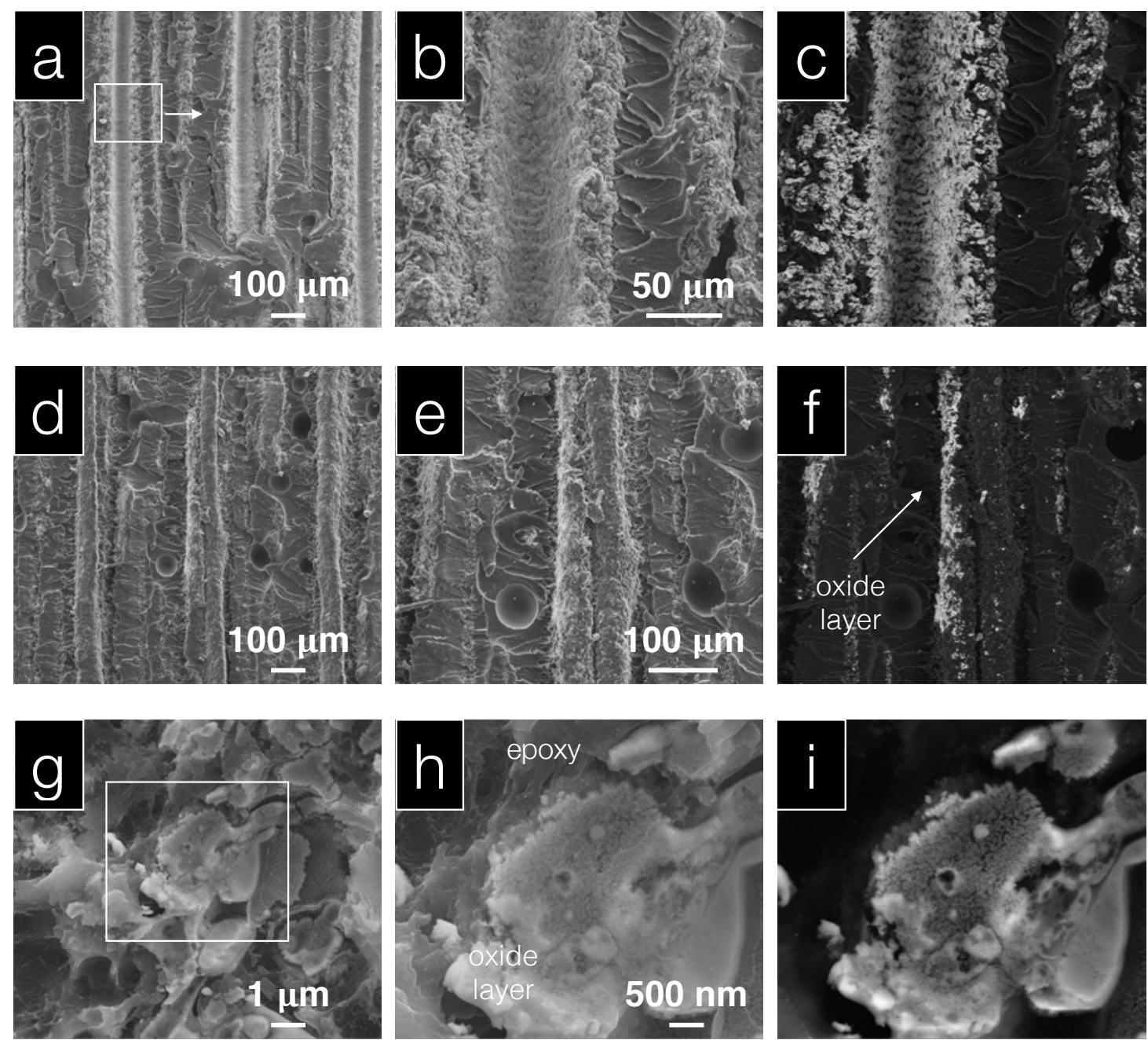

Figure 9: Fractured surfaces of the sample treated at $30 \mathrm{~W}$ and $250 \mathrm{~mm} / \mathrm{s}$. (a),(b): Typical appearance of adhesive interlocked within the cavity created by the ablation process. (c): Back scattered version of (b). (d),(e): Fracture surfaces of the mating substrate. (f): Back scattered version of (e). (g) and (h): high resolution imaging of typical island of surface oxide decohered from the substrate during sample debonding. (i): back scattered version of (h). For all figures: crack propagation was from left to right. 


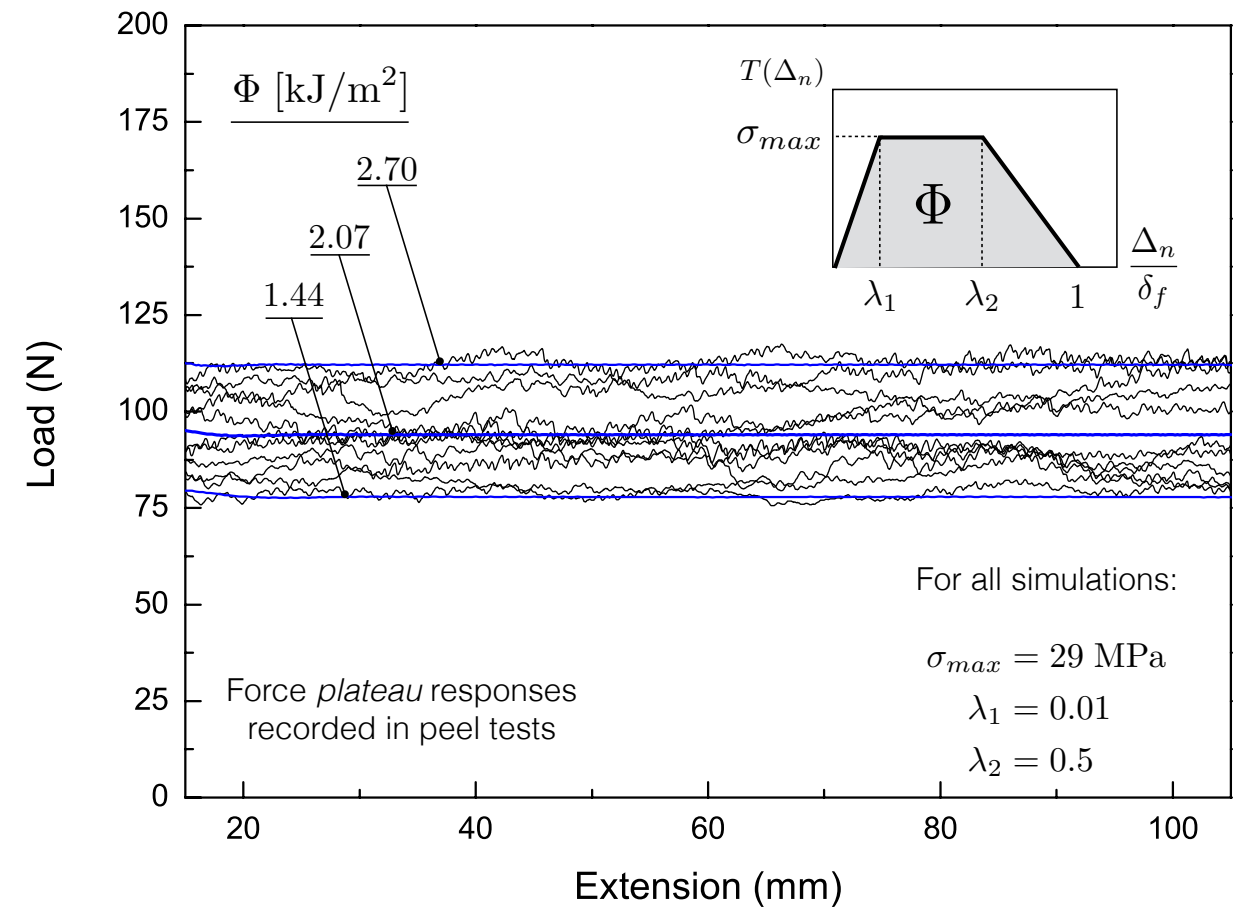

Figure 10: Comparison among experiments and simulations in the plateau region of the global response pertaining to laser treated samples in air at $250 \mathrm{~mm} / \mathrm{s}$. The insert shows the traction separation relation employed in the simulations along the main input parameters. 


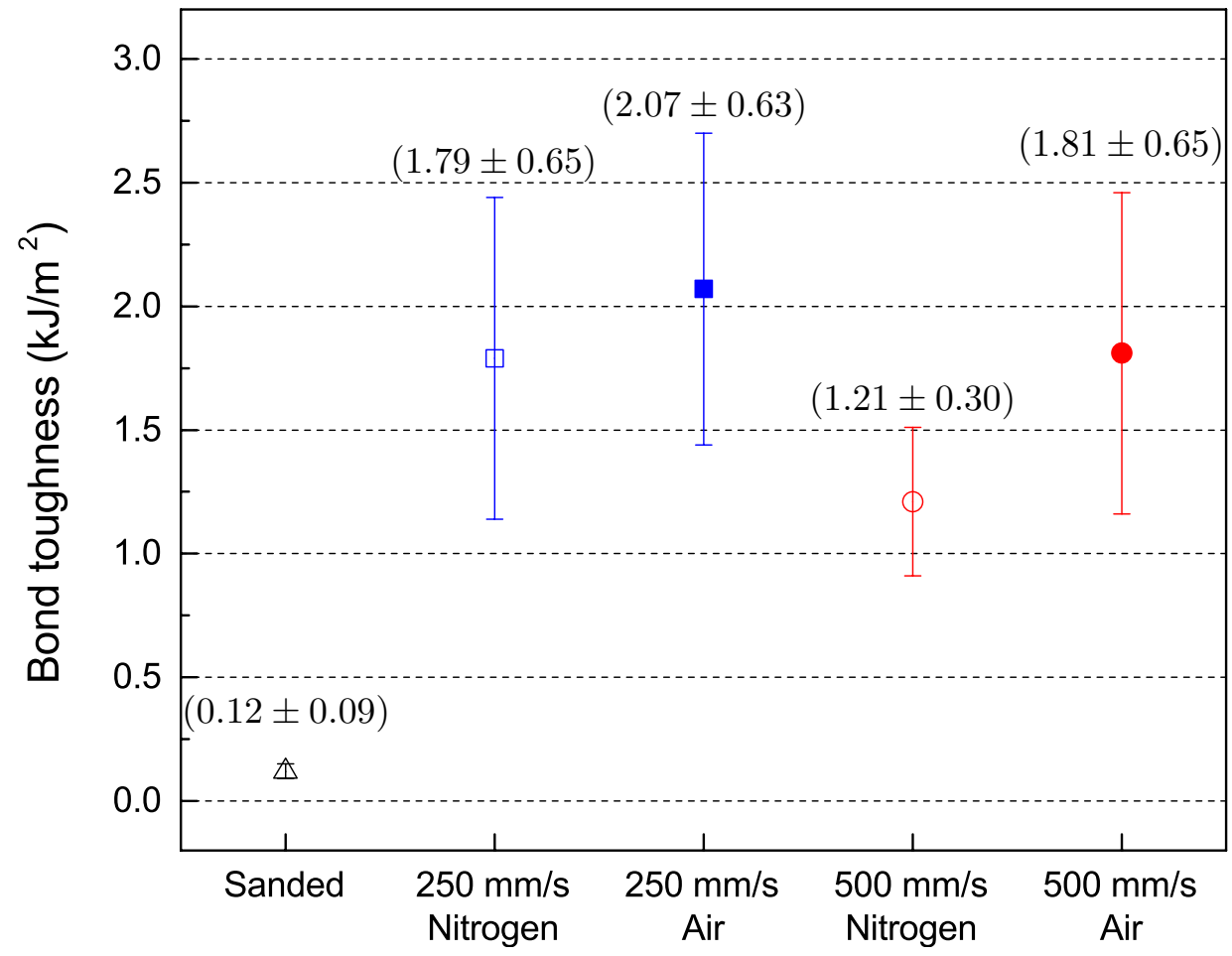

Figure 11: Bond toughness extracted from finite element simulations pertaining to the selected laser processing conditions. 